\section{P51 Portion Distortion Degree (PDD) Scale: Development, Validation and Applications}

Catalina Velez, MS, catalina.velez@gmail.com, Cornell University, 112 Warren Hall, Cornell University, Ithaca, NY 14853; Brian Wansink, PhD, Cornell University

Objective: To develop a scale that combines all the drivers of portion distortion into one. Since literature has shown various ways that portion distortion may be expressed in individuals (causing future eating problems including overweight and obesity), this research can provide new ways to for public programs to detect causes of obesity and encourage productive weight loss.

Study Design, Setting and Participants: Item generation was made using in-depth interviews with 8 participants recruited through snowball sampling. Scale dimensionality and internal consistency (exploratory factor analysis) and scale confirmation (confirmatory factor analysis) was performed with 126 participants recruited from mTurk for monetary compensation. At the end of the survey portion choice (Subway sandwiches: Footlong, six inches) was used to measure current portion distortion.

Outcome, Measures and Analysis: Proposed PDD scale was tested for internal consistency, dimensionality, and criterion validity. Exploratory factor analysis (EFA) using principal axis factoring (PAF) with varimax rotation in SPSS. Afterwards confirmatory factor analysis (CFA), using AMOS with maximum likelihood estimation provided construct and discriminant validity tests. Subsequently PDD scale was tested using a logistic regression determining PDD effect on portion choice.

Results: Four dimensions of the scale were found, all with Cronbach alpha $>0.8$, CFA shows successful goodness of fit (RAMSEA $=0.065, \mathrm{CMIN} / \mathrm{DF}=1.53)$. Results of the logistic regression showed individuals who choose smaller portions have significantly less PDD ( $\mathrm{p}>0.01)$.

Conclusions and Implications: PDD scale reaffirm that portion distortion is driven by different factors and not only economic utility. Furthermore, the usage of the PDD scale can help to measure individual proneness to portion distortion and asses its obesity risk.

Funding: None

\section{P52 A Systematic Review of U.S. Food Retailers' Perspectives Using Behavioral Economic Domains, 1980 to 2016}

BaileyHoughtaling, MS, RD, baileyh@vt.edu, Virginia Polytechnic Institute and State University, Wallace Hall, 295 West Campus Drive, Blacksburg, VA 24061; Elena Serrano, PhD, Virginia Polytechnic Institute and State University; Vivica Kraak, PhD, RD; George Davis, PhD; Sarah Misyak, PhD

Objective: Understanding the economic opportunities and barriers from the retailer (i.e., owners, managers, and employees) perspective to support effective partnerships and financial stability of involved businesses is imperative in order to inform health promotion interventions targeted at low-income consumers, and is a scope lacking in current food environment research. To examine food-retailer perspectives respective to choice architecture and marketing strategies used to encourage healthy food purchases, in order to understand factors that influence intervention feasibility and effectiveness.

Study Design, Setting, Participants: A systematic literature review using the Preferred Reported items for Systematic Reviews and Meta-Analysis guidelines. United States food environment research inclusive of retailer perspectives from years 1980-2016 were included per review criteria.

Outcome Measures and Analysis: Perspectives were categorized using eight behavioral economic strategies (place, profile, portion, pricing, promotion, priming, prompting, and proximity). Co-investigators reviewed the outcomes data and the U.S. Department of Agriculture's nutrition evidence library checklist for study quality was used.

Results: Of included articles $(n=22)$, most perspectives describe factors that may influence the practical application of behavioral economic strategies such as community and/or economic context of food-retail store, interpersonal or community relationships, store or supplier policies, space and structures, and the convenience, demand, profitability, and shelf-life of unhealthy products. A smaller results subset describes beneficial strategies implemented, such as taste promotions or food displays, from the retailer perspective.

Conclusions and Implications: Researchers need be cognizant of opportunities and barriers specific to the implementation of behavioral economic strategies in the food-retail context in order to design sustainable and realistic interventions to influence consumer healthy food purchases.

Funding: Supplemental Nutrition Assistance Program Education

\section{P53 Association Between Food Shopping Motivators and Home Food Environment}

Abiodun T. Atoloye, MSc, abiodun.atoloye@aggiemail.usu. edu, Utah State University, 8700 Old Main Hill, Logan, UT 84322; Carrie Durward, PhD, RD, Utah State University

Objective: This study examines whether the home food environment is related to food shopping motivating factors such as taste, cost, convenience, weight control, and nutrition.

Study Design, Settings, Participants: This is a secondary analysis of a cross-sectional survey $(n=123)$ of Supplemental Nutrition Assistance Program (SNAP) recipients.

Outcome Measures and Analysis: Participants were surveyed about their home food environment, factors

Continued on page $S 44$ 